\title{
EXPERIMENTAL INVESTIGATIONS OF PLANAR WATER SHEETS FLOWING UNDER GRAVITY
}

\author{
AMINE KACEM ${ }^{1}$, YVES LE GUER ${ }^{1}$, KAMAL EL OMARI $^{1} \&$ PASCAL BRUEL $^{2}$ \\ ${ }^{1}$ Univ Pau \& Pays Adour, Laboratoire des Sciences de l'Ingénieur Appliquées la Mécanique et au Génie Électrique \\ - Fédération IPRA, Pau, France \\ ${ }^{2}$ CNRS/Univ Pau \& Pays Adour, Laboratoire de Mathématiques et de leurs Applications de Pau - Inria Cagire \\ Team - Fédération IPRA, Pau, France
}

\begin{abstract}
In this work, the main physical characteristics of plane water sheets flowing under gravity and surrounded by free air are experimentally investigated. By varying the mean flow rate through a $0.8 \mathrm{~mm}$ $\times 100 \mathrm{~mm}$ nozzle, water sheets are produced over a range of Reynolds and Weber numbers between 210 to 1240 and 0.37 to 13.52 , respectively. First, the sensitivity of the sheets shapes to a mass flow rate variation is evidenced. For a sufficiently high flow rate, a liquid sheet forms with two rims bordering it. These rims join after a certain length $L_{c}$ resulting in a triangle-like shape of the sheet. This characteristic length is compared with a theoretical prediction given by a model valid for $R e \gg 1$. Furthermore, some capillary waves forming a striped pattern are present at the sheet interface near the rims and are propagating towards the central axis as the sheet falls. These waves are interpreted as the consequence of the displacement of a high curvature gradient zone at the rim-sheet interface as suggested by their stationary shape. The critical mass flow rate at which the sheet destabilization is systematically observed is $Q_{c}=0.056 \mathrm{~kg} . \mathrm{s}^{-1}$. It corresponds to a Weber number $W e \simeq 2.7$, a value in line with the theoretical one $W e_{t h}=O(1)$ which usually indicates a sufficient condition to maintain a stable sheet. Such ruptures are characterized by the appearance of expanding hole(s), predominantly in the lower half of the sheet. The experimentally determined mean expansion velocity proved to be within $\pm 20 \%$ of that provided by the well-known Culick expression. As expected, when considering mass flow rates below the above mentioned critical one, an intermittent regime of rupture is obtained characterized by the presence of sheets, threadlines, jets or drops.
\end{abstract}

Keywords: liquid sheets, instabilities, holes, capillary waves.

\section{INTRODUCTION}

The present study is originally motivated by the enrichment of the understanding of curtain dynamics and stability in the coating context where viscous sheets usually flow under gravity at low flow rates. Indeed, liquid sheets or curtain flows have been extensively studied because of their theoretical and technological interest. For instance, coating process for plywood and paper manufacturing can be seen as common examples where the aim is to maintain as stable a curtain as possible. In this work, we are interested in the dynamics and the shape of thin water sheets flowing under gravity at moderate flow rates. Brown [1] was one of the first to study experimentally the behaviour of a plane liquid curtain impinging on a moving surface. He found that a minimum liquid flow rate is required to maintain the stability of the liquid sheet. By balancing the inertia forces and the surface tension which traduced the equilibrium of a free edge, Brown [1] deduced a stability criterion for the sheet based on the Weber number $W e_{c}=\frac{\rho U^{2} h}{2 \sigma}=1$ ( $\rho$ is the liquid density, $h$ the local curtain thickness, $U$ the local fluid velocity and $\sigma$ the surface tension).

Lin [2] has found another interpretation of the Weber number. He applied a spatial stability analysis and deduced that $W e$ is the ratio between the fluid velocity and the sinuous wave group velocity. Thus, he concluded that the condition $W e>1$ guarantees the sheet stability since any disturbance will be swept downstream with the flow of the liquid curtain and will 
(a)

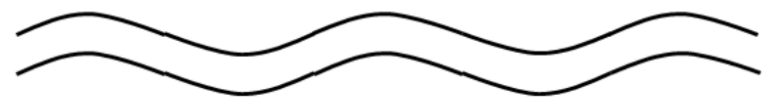

(b)

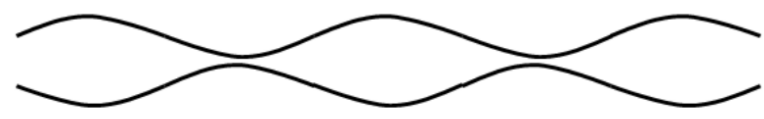

Figure 1: Sinuous wave (a) and varicose wave (b).

not propagate upstream. On the other side, the sheet was shown to be temporally and spatially stable to a varicose (symmetrical) disturbance (see Fig. 1).

Later on, most of the experimental studies concerned liquid sheets flowing at high Reynolds and Weber numbers. Nevertheless, in the last two decades, plane jets falling freely under the effect of gravity for low flow rates have regained interest. Finnicum et al. [3] reported observations of stable liquid curtains in a wide range of flow conditions, including $W e<1$. They attributed the observed stability at low We to nonlinear effects that may stabilize disturbances after they grow to a finite amplitude. In [4], the effects of surface-active agents present in the water sheets have been analyzed systematically and various flow regimes under different flow rates were identified. Roche et al. [5] studied experimentally the response of a liquid sheet near the break-up limit to localized perturbations introduced with a thin needle touching the curtain and they showed that holes do not necessarily expand for $W e<1$. Lhuissier et al. [6] studied the response of a steady free-falling liquid curtain perturbed by focused air jets blowing perpendicularly against it. For steady symmetrical air jets perturbations, the local curtain thickness was shown to be changed allowing the emergence of zones with a local Weber number decreasing in the direction of the flow i.e. $\frac{\partial W e}{\partial x}<0$. As a consequence, a steady hole forms downstream. Furukawa et al. [7] measured the minimum flow rate $Q_{c}$ compatible with a stable curtain formation for polyester resin solutions and found that $Q_{c}$ decreased with increasing viscosity of the liquids.

In the present work, we experimentally investigate planar water sheets flowing with flow rates of the same order of the one needed to maintain a stable sheet. Thus, the studied cases correspond to a Weber number $W e$ which is $O(1)$. However, the water sheets Reynolds numbers $R e$ were always $\gg 1$ which makes the principal difference with respect to viscous sheets flowing near the breakup regime where $R e=O(1)$.

In section 2, the experimental setup is presented with a brief description of the visualization technique used. Then, in section 3, the experimental results obtained for the dynamics of the water sheets are presented and discussed.

\subsection{Flow loop and nozzle}

\section{EXPERIMENTAL SETUP}

The plane water sheets of the present study have been produced with the apparatus shown in Fig. 2. It consists of four main parts: the headbox where a convergent nozzle is mounted, the dump tank, the overflow tank, and a volumetric pump. A sketch of the nozzle geometry is also given in Fig. 2. The nozzle height is $150 \mathrm{~mm}$ with a converging rectangular cross section (contraction ratio of 38.75:1). At the exit, the nozzle aperture has a $0.8 \mathrm{~mm} \times 100 \mathrm{~mm}$ rectangular cross-section. The flow rate was adjusted with a diaphragm valve positioned 

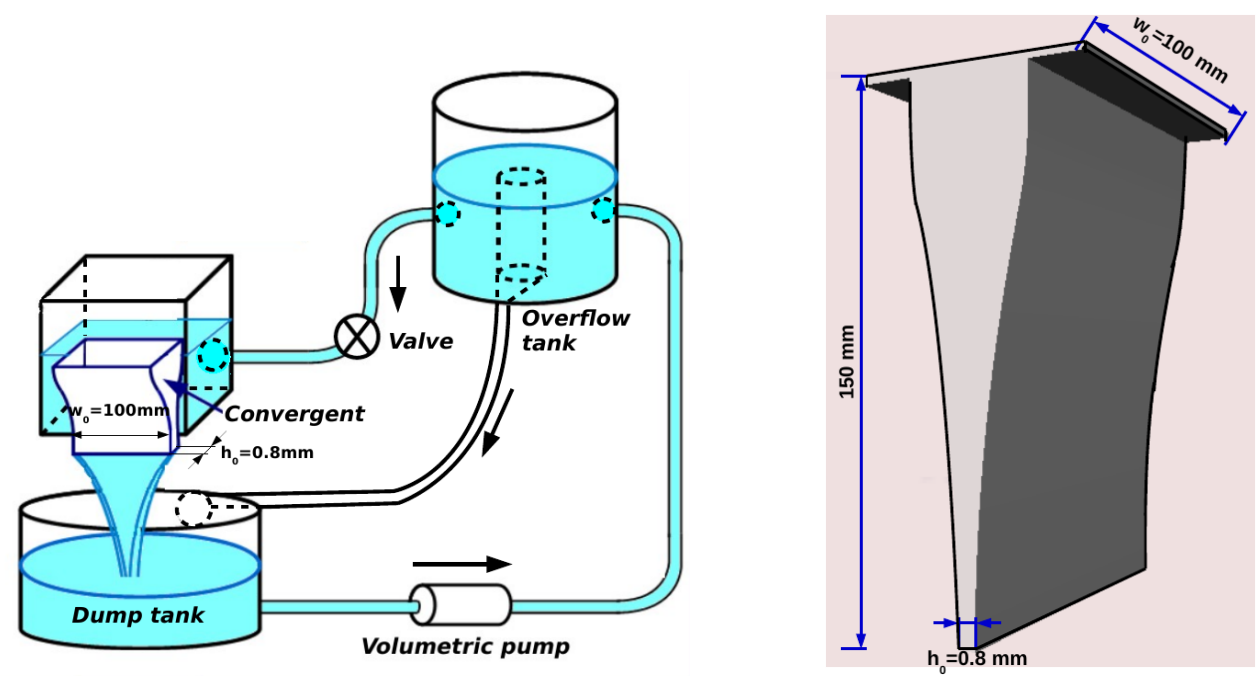

Figure 2: Experimental apparatus: the flow loop (left) and the nozzle sketch (right).

downstream of the overflow tank. Each flow rate corresponds to a fixed water height in the nozzle. The flow rate was determined by weighing collected liquid in a container during a given period of time. Such a procedure was repeated five times for each configuration. A particular attention was paid to check that the flow rate does not fluctuate too much around its mean value (typically $1 \%$ of water height fluctuation). The water temperature was $22 \pm 2{ }^{\circ} \mathrm{C}$, which corresponds to a kinematic viscosity of $v=0.9810^{-6} \pm 0.0510^{-6} \mathrm{~m}^{2} . \mathrm{s}^{-1}$, a surface tension of $\sigma=0.0724 \pm 0.0004 N . \mathrm{m}^{-1}$ and a density of $\rho=995.8 \pm 0.5 \mathrm{~kg} . \mathrm{m}^{-3}$.

\subsection{Flow visualisation}

The shadowgraph method was used to visualize the plane jet which was uniformly illuminated by a $180 \mathrm{~mm} \times 200 \mathrm{~mm}$ backlight Leds screen $(470 \mathrm{~nm})$. A CMOS Sentech video camera with a maximum frequency of 340 frames per second and equipped with a Nikon lens with a focal length of $50 \mathrm{~mm}$ was used to take pictures of the water sheet. This frame rate was sufficient to capture the dynamics of the expanding holes. Last but not least, this technique is quite efficient to detect accurately the shape of the rims, the capillary waves and the sheet geometry.

\section{RESULTS}

This section is composed of four main parts: In the first one, we show the dependence of the sheet geometry on the flow conditions. In the second part, we explain the appearance of ripples or capillary waves on these sheets and we measure their propagation velocity. The third part is dedicated to the investigation of the transition from a stable sheet to an unstable one. In the last part, a qualitative description of other flow regimes associated with very low flow rates is given. 

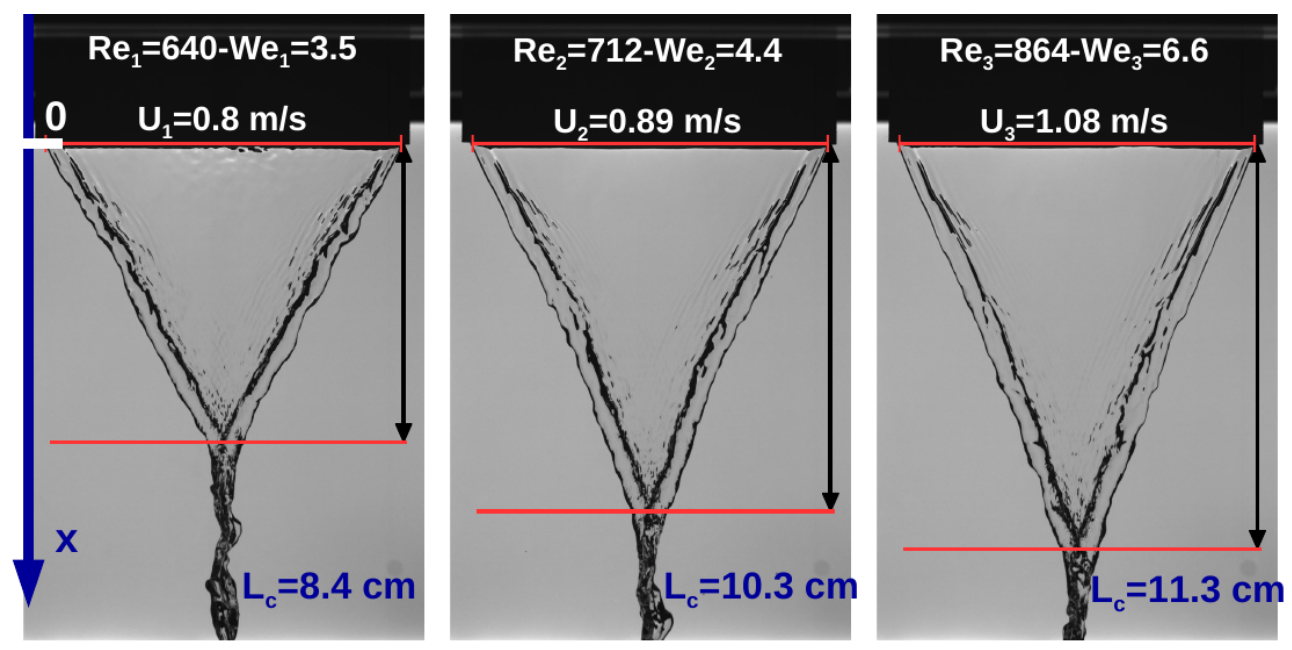

Figure 3: Influence of the flow rate increase on water sheet configuration. The mean velocity and the dimensionless numbers were calculated on the basis of the measured flow rate and the liquid sheet dimensions at the nozzle exit.

\subsection{Water sheets geometry}

Fig. 3 illustrates the different shapes of the water sheet obtained for three different flow rates. The competition between capillary retraction (surface tension acting on the bordering rims) and the flow inertia results in different shapes and different convergence lengths $L_{c}$.

As a result of the surface tension action on the rims, the width of the sheet decreases from the nozzle exit to the point of rims convergence. As the liquid flow rate is increased, the height of the sheet increases. The relatively high sheet velocity in these cases delays the effect of surface tension; therefore, the convergence area appears farther downstream. The simple theoretical model proposed by Chubb et al. [8] is used to estimate $L_{c}$ and the spatial evolution of the sheet width $w(x)$, where $x$ is the vertical flow direction coordinate. This model is based on a balance between inertia, capillarity and gravity and is valid for $R e \gg 1$. More details can be found in [9]. The theoretical width $w(x)$ and the convergence length $L_{c}$ of the sheet are found to be:

$$
\begin{aligned}
\frac{w(x)}{w_{0}} & =1-\frac{2 F r_{0}}{3} \sqrt{\frac{8}{W e_{0}}}\left[\left(1+\frac{x}{F r_{0} w_{0}}\right)^{3 / 4}-1\right] \\
\frac{L_{c}}{w_{0}} & =\frac{F r_{0}}{2}\left[\left(1+\frac{3}{2 F r_{0}} \sqrt{\frac{W e_{0}}{8}}\right)^{4 / 3}-1\right]
\end{aligned}
$$

Where $F r_{0}=\frac{U_{0}^{2}}{g w_{0}}$ is the Froude number based on the nozzle width $w_{0}, W e_{0}=\frac{\rho U_{0}^{2} h_{0}}{2 \sigma}$ is the Weber number based on the nozzle thickness $h_{0}$ and $U_{0}$ is the mean velocity at this exit.

In Figs 4 and 5, the water sheets shapes obtained experimentally as well as the measured lengths $L_{c}$ are compared with their theoretical counterparts. The relative difference between $L_{c, \exp }$ and $L_{c, \text { theo }}$ is below $15 \%$. This slight discrepancy can be explained by the consideration of an idealized acceleration of the vertical component of velocity in the model 


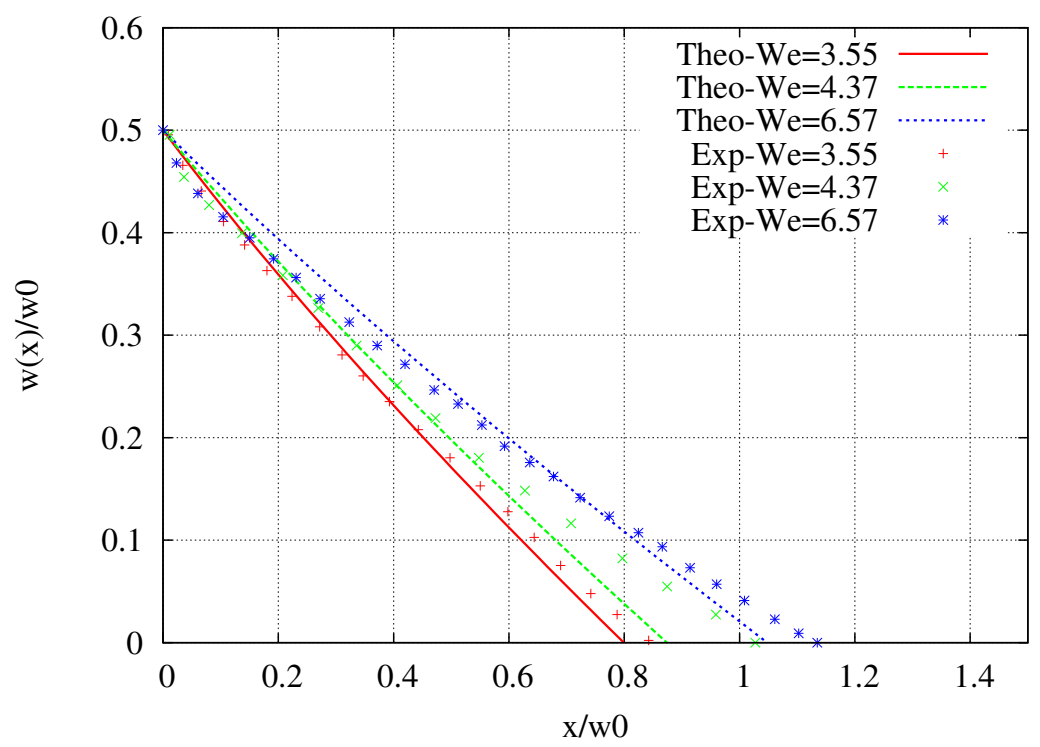

Figure 4: Comparison between theoretical and experimental results: streamwise evolution of the sheet width $w(x)$ along the vertical direction $x$.

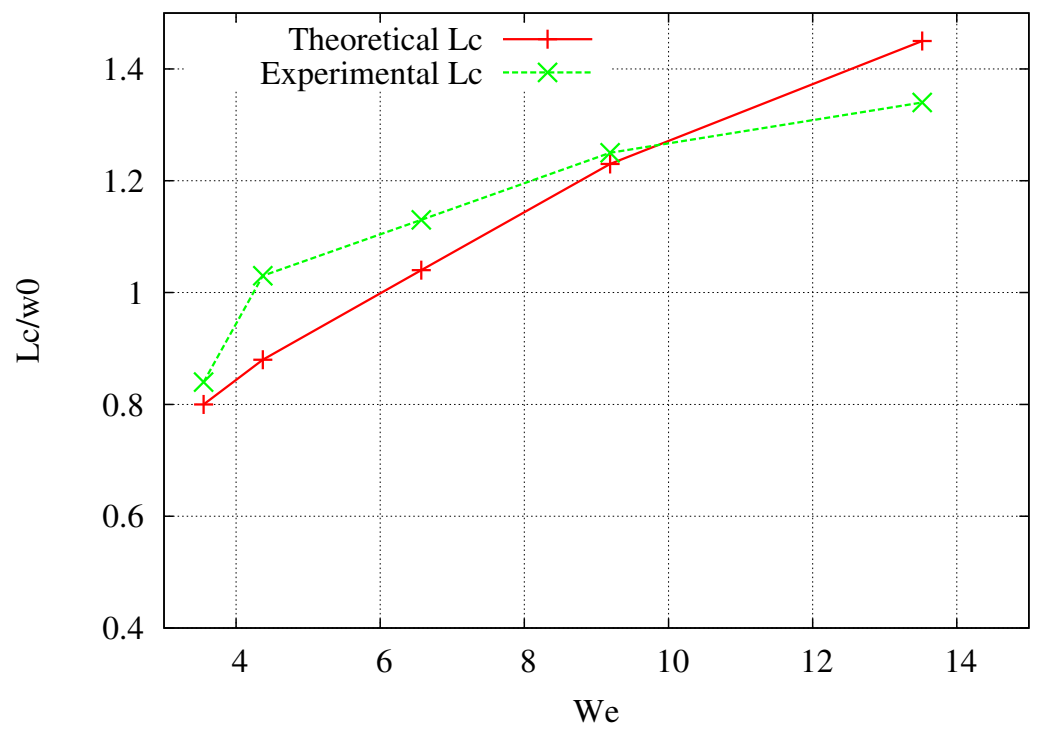

Figure 5: Comparison between theoretical and experimental results: dependence of the convergence length $L_{c}$ on the Weber number. The different Weber numbers were obtained by varying the water mass flow rate. 


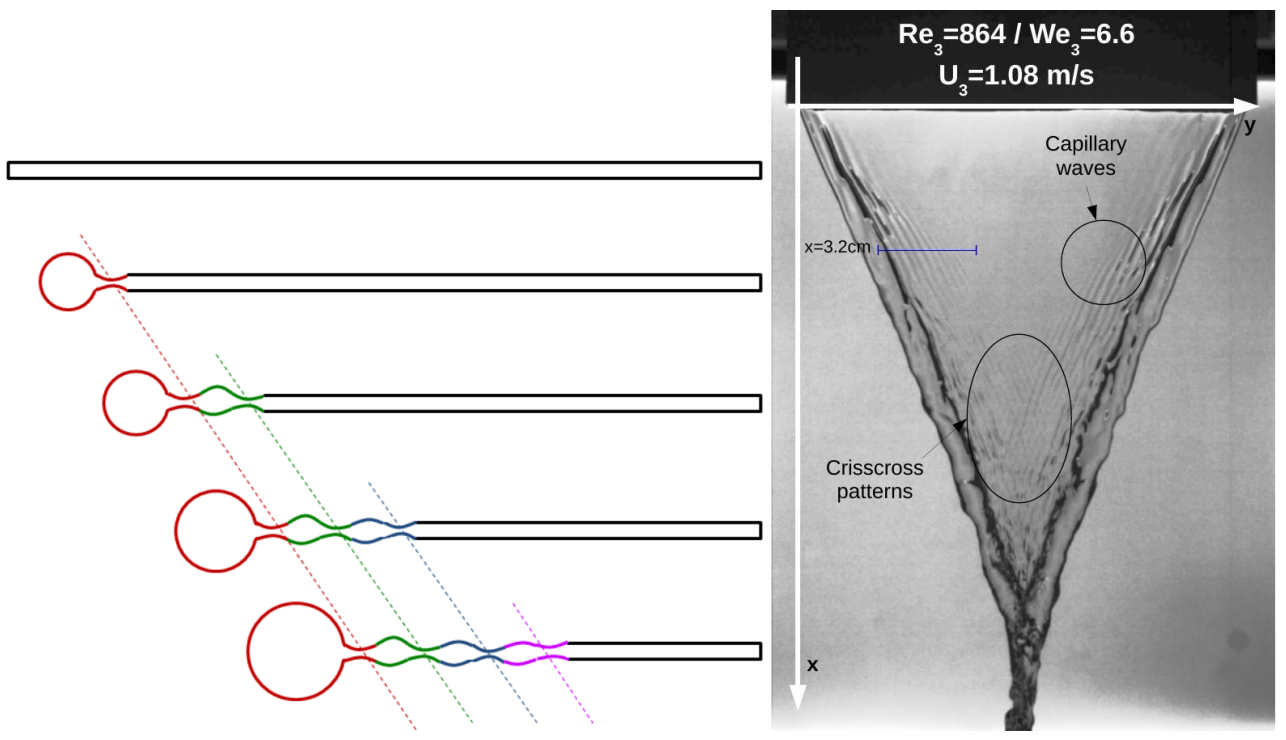

Figure 6: Sketch of 2D capillary retraction in the case of $O h \ll 1$ (left) and capillary ripples on the water sheet for $R e=864$ (right).

$u=\sqrt{U_{0}^{2}+2 g x}$ which ignores small viscous effects. Another factor that might be a source of discrepancy is the modeling of the curvature of the rim in a plane perpendicular to the gravity direction.

\subsection{Capillary waves}

By enhancing the contrast of a liquid sheet photography as in Fig. 6 (right), we observe clearly striped patterns indicating the presence of surface capillary waves. Those ripples present in the transition area between the rims and the planar part of the sheet result from a competition between viscous and surface tension forces. The Ohnesorge number which specifies the ratio between capillary and viscous forces is $O h=\frac{\mu}{\sqrt{2 h \rho \sigma}}=0.003 \ll 1$. This low value in our case (water-air system) indicates that capillary waves will take place near the rims as indicated in $[10]$.

We sketch in Fig. 6 (left) the 2D process of a capillary wave development with time, combined with the lateral sheet retraction, in the case of an initially rectangular liquid film for a small Ohnsorge number [11]. The temporal evolution of this liquid film can be seen as a $2 \mathrm{D}$ approximation of the spatial evolution of a horizontal section of the sheet along the vertical axis $x$. In this case, successive crests are created while being horizontally displaced towards the unperturbed part of the liquid film due to the rim retraction. The rim retraction velocity value tends to the well-known Culick velocity [12] $u_{c}=\sqrt{\frac{2 \sigma}{\rho h_{0}}}$. However, in our three dimensional case, there is a downstream gravity driven-flow superimposed to the capillary rim lateral retraction. As a result, a given crest is moving with an additional vertical velocity component which can be estimated by the free fall law $u=\sqrt{U_{0}^{2}+2 g x}$. Immediately after the rim formation, a first crest is created by capillarity in the rim region characterised by a brutal change in curvature. In Fig. 6 (left), the mechanism by which, once created, a crest 
is advected with the rim velocity is illustrated. These crests are schematically tracked with dotted lines. When gravity comes into play, crests are translated approximately parallel to the rim and this lead to the appearance of dark stationary inclined lines as can be seen in Fig. 6 (right). By determining the rim axis slope and using the free fall approximation for the vertical velocity, the retraction velocity can be calculated. The value $u_{\text {retraction }} \simeq 0.59 \mathrm{~m} . \mathrm{s}^{-1}$ is obtained which corresponds also to the horizontal displacement of capillary waves after their emergence while falling vertically.

As the first capillary crest is created and in a reference frame moving with $u_{\text {retraction }}$, the region corresponding to a high curvature gradient moves towards the sheet axis and a second crest appears by capillarity. New crests continue to appear downstream by the same process until waves from each rim interfere with each other resulting in a crisscross pattern (Fig. 6 (right)). The rate of creation of new crests can be associated to a velocity that we called $u_{\text {crest }}$. This velocity is related to the horizontal distance separating the tips of two successive dark lines. By making an equivalence between velocities and distances on the sheet we deduced that $u_{\text {crest }} \simeq 0.48 \mathrm{~m} . \mathrm{s}^{-1}$. We note that the two calculated velocities $u_{\text {retraction }}$ and $u_{\text {crest }}$ are of the same order of the Culick velocity $u_{c}=0.42 \mathrm{~m} . \mathrm{s}^{-1}$.

In [13], the authors interpreted the capillary instability as a Kelvin-Helmoltz one. They related the waves to the strong shear layer created by the liquid retraction in the surrounding gas. Such an explanation is not in line with the present observations evidencing stationary waves i.e. not growing in time. Another argument is that the liquid in the sheet center is subject to a vertical movement with a magnitude of the same order as the lateral one and no ripples are created in this zone.

As mentioned previously, the contrast was enhanced in Fig. 6 (right), thus crests and troughs are more clearly distinguishable. They are characterised by local minimums and local maximums of Grey Levels (GL) due to the presence of high and low curvatures (convex and concave), respectively. Analysing GL on these images with a dedicated software gave access to the determination of the horizontal gap between two successive capillary waves. The wavelength was found to vary from $\lambda_{0}=1.7 \mathrm{~mm}$ to $\lambda_{0}=2.1 \mathrm{~mm}$. Qualitatively, according to the GL analysis, the amplitude of these capillary waves peaks near the rim and then decays toward the center (see Fig. 7).

A common physical parameter used to describe flows involving strong capillary effects is the capillary length $l_{c}$ which is given by: $l_{c}=\sqrt{\frac{\sigma}{\rho g}}=2.7 \mathrm{~mm}$. This may be interpreted in our case as a characteristic length in the vicinity of the nozzle exit corners (where the liquid inertia can be considered small compared to surface tension and gravity effects). This capillary length is of the same order of magnitude as the initial diameter of the rim $D_{\text {rim }}=3.6, \mathrm{~mm}$ (where the liquid is already leaving the nozzle). However, there is another interesting length scale $l_{v c}=\frac{\mu^{2}}{2 \sigma \rho}=6.5 \mathrm{~nm}$ which corresponds to the distance over which there is an equilibrium between capillary and viscous effects. This length can be easily connected to the Ohnesorge number through: $O h=\sqrt{\frac{l_{v c}}{h_{0}}}$ (where $h_{0}$ is the liquid sheet thickness at the nozzle exit) and may be interpreted as the length scale over which capillarity can act on the free surface of a viscous sheet. When $O h \ll 1$, as in our case, we have $l_{v c} \ll h_{0}$, so capillary waves can easily manifest on the free surface as in our case.

\subsection{Transition between stable and unstable water sheets}

In many industrial applications, a stable liquid sheet is sought even at low flow rates. However, searching the minimum flow rate allowing a stable curtain is not trivial. In fact, we noticed that there is a hysteresis phenomenon similar to that mentioned in [14] which 


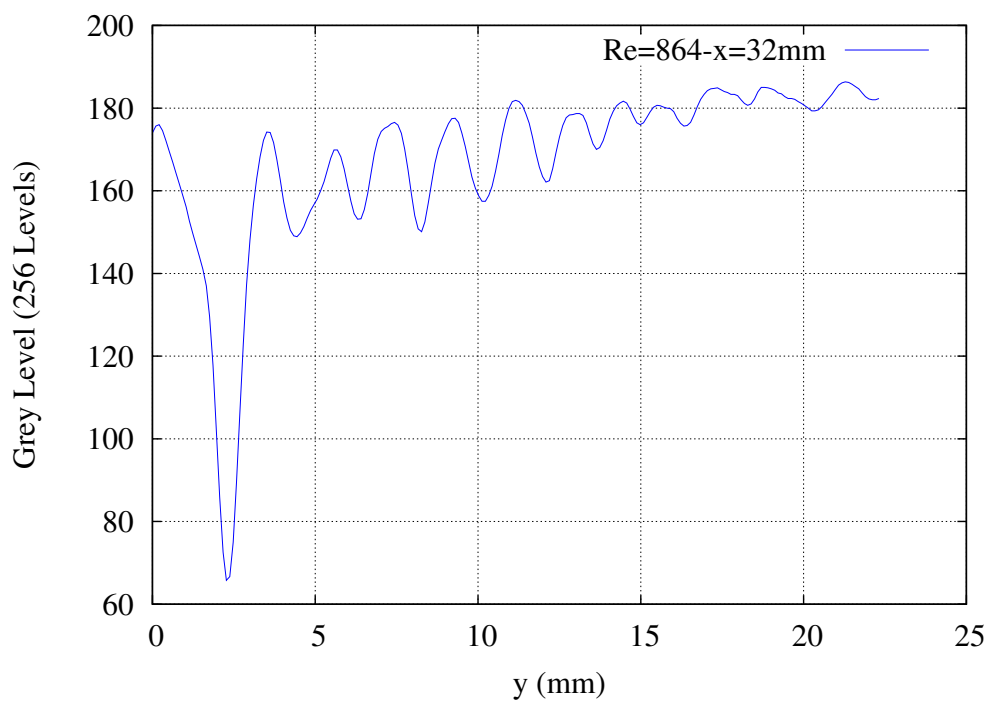

Figure 7: Grey Level variation along a line located at $x=3.2 \mathrm{~cm}$ from the nozzle exit (see Fig. 6 (right)).

may permit to decrease the total flow rate required for the sheet stability. The minimum flow rate for stable curtain was determined by the same procedure as in [14]. First, we delivered a high enough flow rate such that a stable curtain was formed. After that, we lowered gradually the flow rate until the appearance of the first expanding holes on the sheet. We note that in our experiments with water, even with the hysteresis phenomenon, partial ruptures occur before achieving the theoretical condition of $W e<1$. In fact, expanding holes appeared as soon as the mass flow rate was reduced down to $Q_{c}=0.056 \mathrm{~kg} . \mathrm{s}^{-1}$ whereas the theoretical flow rate which corresponds to the condition given by $W e=1$ is $Q=0.034 \mathrm{~kg} . \mathrm{s}^{-1}$. At the critical flow rate, small holes may appear predominantly in the lower thinner half of the sheet. As it is illustrated in Fig. 8 which displays the time evolution of the sheet, a hole expands in all directions including the upstream direction of the flow while being advected and stretched towards the convergence area of the rims. The expansion velocity of the hole was determined by post-processing image series of the sheet. A spatio-temporal diagram was achieved over an horizontal line intersecting the hole (there is no gravity contribution to the hole expansion in this direction). The obtained value is $u_{\exp }=0.58 \mathrm{~m} . \mathrm{s}^{-1}$. We recall that the value given by the Culick's formula [12] is $u_{c}=\sqrt{\frac{2 \sigma}{\rho h_{0}}}=0.42 \mathrm{~m} . \mathrm{s}^{-1}$. This over-prediction indicates that there is a thinning of the sheet which is not taken into account by the Culick's formula. Identifying the measured hole expansion velocity as the Culick one, which is reasonable in our case since $O h \ll 1$, gives an estimate of the local liquid sheet thickness of $h \simeq 0.5 \mathrm{~mm}$.

\subsection{Other flow regimes}

Decreasing further the flow rate value leads to the appearance of different flow regimes as it was the case in the study of Lucas and Meola [4]. First, the two bordering rims detach from the sheet and form two independent jets enframing a smaller sheet for which new rims form. As the flow rate is reduced, the size of the central sheet decreases until it is replaced by a 


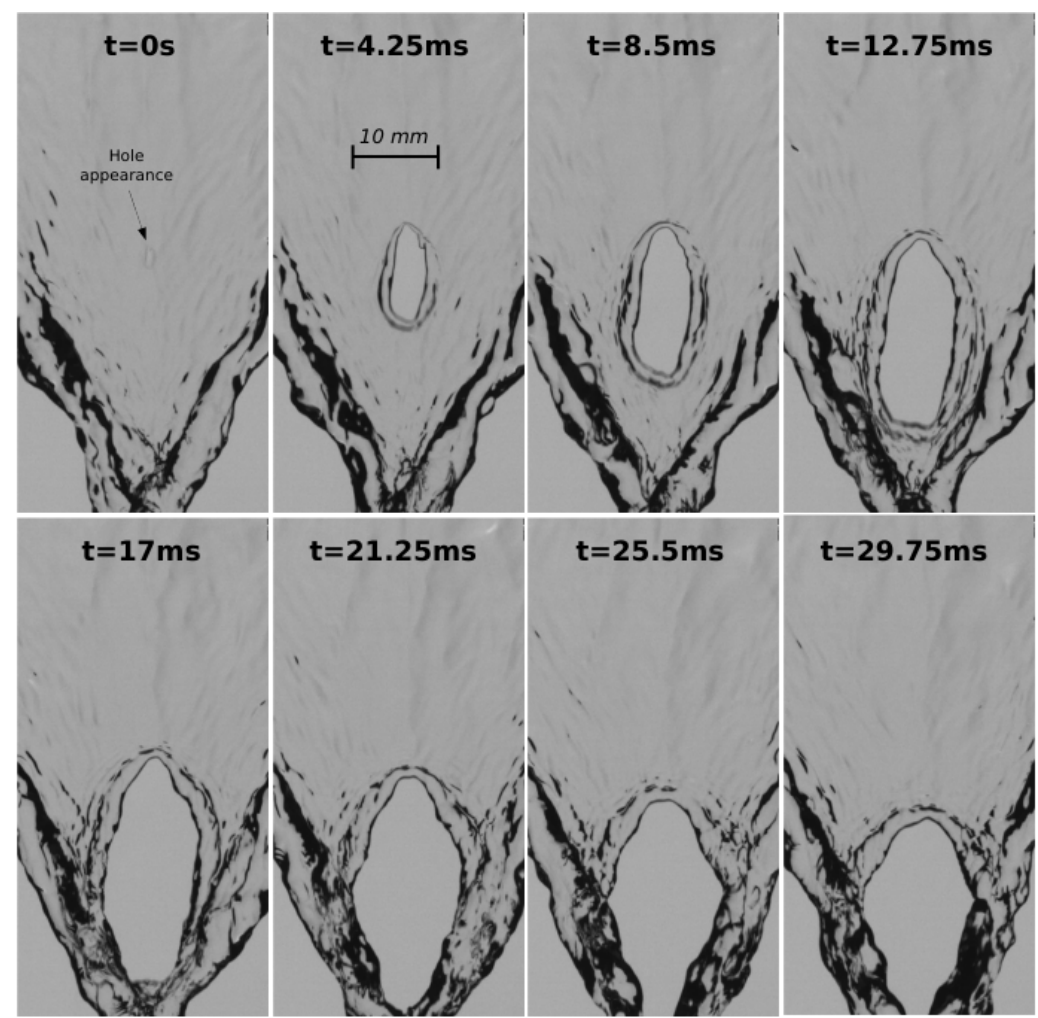

Figure 8: Successive snapshots of an expanding hole for $R e=560-W e=2.7(4.25 \mathrm{~ms}$ between two consecutive images).

simple jet (see Fig. 9). The three-cylindrical-jet feature of the Rayleigh-Plateau instability can be recognized with the water-air interface taking a varicose shape at the source of the observed droplets formation.

\section{CONCLUSION}

The physics of water sheets flowing through a convergent slot nozzle under the action of gravity with moderate Reynolds numbers was experimentally investigated. The main characteristics of these sheets such as the presence of boundering rims, capillary waves and the sheet retraction were analysed and compared to some known theoretical results corresponding to idealized cases. A fair agreement was observed between the experimental shapes and those obtained with an inertial-gravity model. The capillary waves were found to be stationary and it was shown that they have an appearance velocity $u_{\text {crest }}$ in addition to an advection velocity $u_{\text {rim }}$ caused by the surface tension acting on the rims. Moreover, decreasing the flow rate under a critical value $Q_{c}$ leads to the appearance of holes in the lower half of the sheet. The corresponding Weber number $W e_{c}$ was found to be $W e_{c}=2.7>1$ which indicates that the classic stability criteria of $W e>1$ is not always sufficient to maintain a stable sheet. The hole expansion velocity was determined and then injected into the Culick's formula to estimate the sheet thickness in the hole region. For low flow rates, there always exist perturbations of finite amplitude which lead to non linear interaction processes and thus 

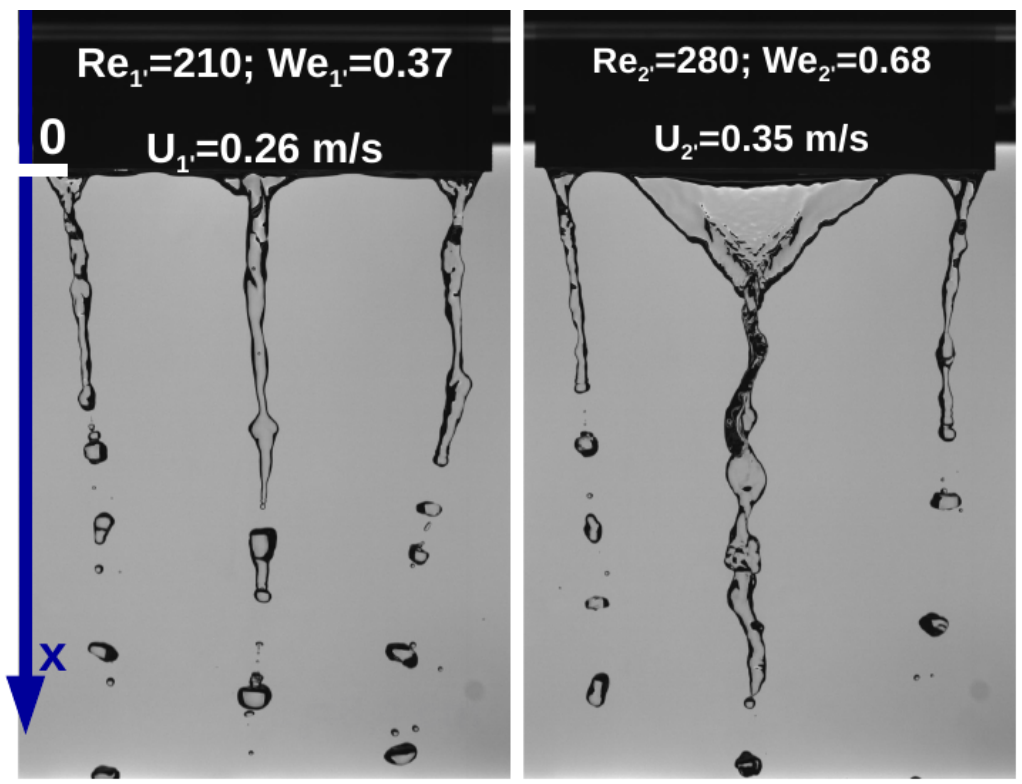

Figure 9: Different regimes observed: jets and droplets (left) and sheet and jets (right).

contribute to the holes emergence. Future investigations will concentrate on the determination of the stability properties in such a case through more quantitative measurements of the thickness variation and the velocity field inside the sheets.

\section{ACKNOWLEDGEMENT}

The authors gratefully acknowledge financial support from the Conseil Général des Pyrénées Atlantiques.

\section{REFERENCES}

[1] Brown, D.R., A study of the behaviour of a thin sheet of moving liquid, J. Fluid Mech., vol. 10, no. 2, pp. 297-305,1961.

[2] Lin, S.P., Stability of a viscous liquid curtain, J. Fluid Mech., vol. 104, pp. 111-118, 1981.

[3] Finnicum, D.S., Weinstein, S.J. \& Ruschak, K.J., The effect of applied pressure on the shape of a two-dimensional liquid curtain falling under the influence of gravity, J. Fluid Mech., 255, pp. 647-665, 1993.

[4] De Luca, L. \& Meola, C., Surfactant effects on the dynamics of a thin liquid sheet, J. Fluid Mech., vol. 300, pp. 71-85, 1995.

[5] Roche, J.S., Le Grand, N., Brunet, P., Lebon, L. \& Limat, L., Perturbations on a liquid curtain near break-up: wakes and free edges, Phys. Fluids, vol. 18, no. 8, 082101, 2006.

[6] Lhuissier, H., Brunet, P. \& Dorbolo, S., Blowing a liquid curtain, J. Fluid Mech., vol. 795, pp. 784-807, 2016.

[7] Furukawa, H., Kanai, H., Nagase, M., Kobayashi, R. \& Alleborn, N., Effect of liquid properties on curtain stability in low flow rate, in ISIJ International, vol. 53, no 1, pp. 119-124, 2013. 
[8] Chubb, D.L., Calfo, F.D., McConley, M.W., McMasters, M.S. \& Afjeh, A.A., Geometry of thin liquid sheet flows, AIAA J, vol. 32, no. 6, pp. 1325-1328, 1994.

[9] Kacem, A., Le Guer, Y., Mejia, A., El Omari, K., Bruel, P., Reynaud, S. \& Grassl, B., Hydrodynamique d'une nappe liquide formée à la sortie d'un convergent, in 22ème Congrès Français de Mécanique, Lyon, 2015.

[10] Brenner, M.P. \& Gueyffier, D., On the bursting of viscous films, Phys. Fluids, vol. 11, pp. 737-739, 1999.

[11] Savva, N. \& Bush, J. W., Viscous sheet retraction, J. Fluid Mech., vol. 626, pp. 211-240, 2009.

[12] Culick, F.E.C., Comments on a ruptured soap film, J. Appl. Phys., vol. 31, no 6, pp. 1128-1129, 1960.

[13] Gordillo, L., Agbaglah, G., Duchemin, L. \& Josserand, C., Asymptotic behavior of a retracting two-dimensional fluid sheet, Phys. Fluids, vol. 23, no. 12, 122101, 2011.

[14] Becerra, M. \& Carvalho, M.S., Stability of viscoelastic liquid curtain, Chemical Engineering and Processing: Process Intensification, vol. 50, no. 5, pp. 445-449, 2011. 